\title{
Elevated human chorionic gonadotrophin levels in a patient with pancreatic carcinoma presenting with a testicular metastasis
}

\author{
H. Taylor, N. Heaton' ${ }^{1}$ P. Farrands ${ }^{1}$, N. Kirkham ${ }^{2}$ and M. Fletcher \\ Departments of Urology, ${ }^{1}$ General Surgery and ${ }^{2}$ Histopathology, Royal Sussex County Hospital, \\ Brighton, East Sussex BN2 5BE, UK
}

\begin{abstract}
Summary: Elevated levels of human chorionic gonadatrophin are associated with primary testicular tumours and usually indicate the presence of trophoblastic elements. Human chorionic gonadatrophin can also be secreted by extragonadal tumours which may metastasize to the testes. A patient is described presenting with a testicular tumour secreting human chorionic gonadatrophin which later proved to be a metastasis from a pancreatic adenocarcinoma. Up to $70 \%$ of malignant pancreatic tumours can secrete human chorionic gonadatrophin.
\end{abstract}

\section{Introduction}

Human chorionic gonadotrophin (HCG) levels are routinely measured in patients presenting with testicular tumours. Elevated levels are associated with primary gonadal tumours, particularly teratomas, but HCG may be produced by extragonadal malignancy.

\section{Case report}

A 77 year old man was admitted with a one month history of weight loss, abdominal pain and a poor appetite. He also complained of a painless, hard swelling in the right hemi-scrotum. On examination he was cachectic, but there was no lymphadenopathy and the abdomen was normal to palpation. There was an irregular $3 \mathrm{~cm}$ by $2 \mathrm{~cm}$ mass in the right testis. The left testis and a rectal examination were normal.

Investigations included a haemoglobin of $9.7 \mathrm{~g} /$ $\mathrm{dl}$ and normal urea, electrolytes, liver function tests, serum alpha-fetoprotein and prostatic acid phosphatase. The serum $\beta$ HCG, however, was raised at $155 \mathrm{IU} / \mathrm{l}$ (normal range $<5 \mathrm{IU} / \mathrm{l}$ ). A chest $\mathrm{X}$-ray showed emphysematous changes only. An ultrasound scan of the right testis revealed a $2 \mathrm{~cm}$ mass typical of a tumour.

A right orchidectomy with a high inguinal tie was performed through an inguinal incision. The histology revealed the tumour to be a secondary

Correspondence: N. Heaton, F.R.C.S., Firm III Office, King's College Hospital, Denmark Hill, Camberwell, London SE5, UK.

Accepted: 31 May 1990 from an adenocarcinoma of intestinal type, possibly from a colonic primary (Figure 1). Immunocytochemical staining of the tumour was positive for carcinoembryonic antigen (CEA) (Figure 2) and negative for prostatic markers.

A colonoscopy was normal, but on duodenoscopy there was a raised mucosal plaque in the second part of the duodenum. Biopsies revealed a moderately differentiated adenocarcinoma infiltrating beneath the mucosa, consistent with an adenocarcinoma of the exocrine pancreas. It had the same histological features as the testicular tumour.

A repeat abdominal ultrasound scan of the pancreas revealed dilatation of the main duct within a hyperechoic gland and a large lesion in the body presumed to be tumour. The serum $\beta$ HCG has remained raised at $180 \mathrm{IU} / \mathrm{l}$. The patient has been treated symptomatically.

\section{Discussion}

Metastatic tumours of the testis are well recognized, but are unusual. The likelihood of metastatic disease as the cause of testicular swelling increases above the age of $50^{1}$ particularly if there is bilateral involvement. The commonest primary tumours to metastasize to the testis include lymphoma, melanoma and carcinoma of the prostate, lung and kidney, ${ }^{1}$ but rarely the pancreas. A raised $\beta$ HCG level in a patient with a testicular tumour usually indicates the presence of trophoblastic elements in a germ cell tumour, but this patient was outside the age range usually associated with teratomas and 


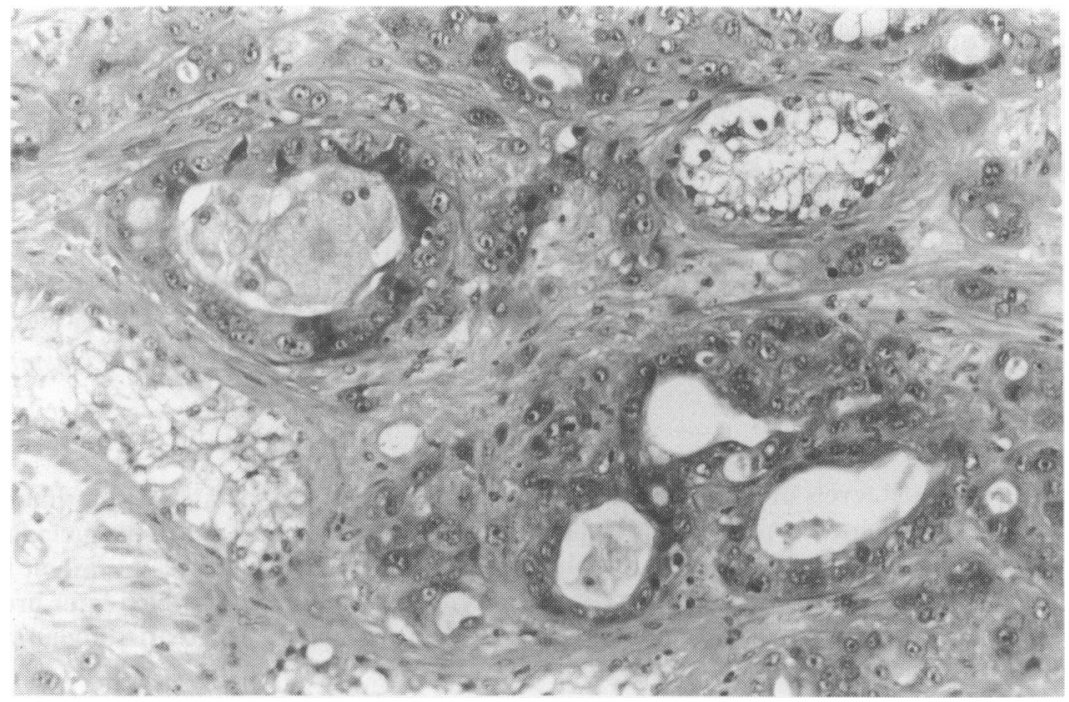

Figure 1 Section of the testis showing the general appearance of the metastatic pancreatic secondary (Haematoxylin 윽 and eosin stain $\times 80)$.

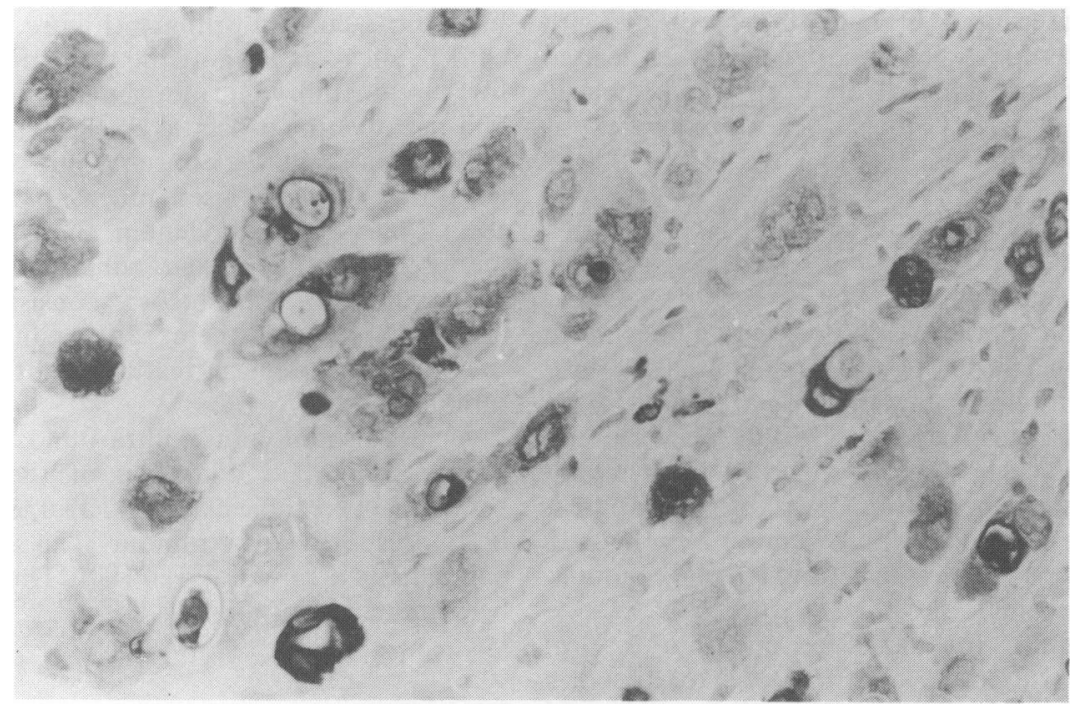

Figure 2 Section of the testis showing HCG-immunoreactivity in areas of metastatic tumour $\times 150$.

seminomas.

HCG is composed of two dissimilar subunits; the alpha subunit shares a common structure with follicular stimulating hormone (FSH), luteinizing hormone (LH) and thyroid stimulating hormone (TSH), and the beta subunit which confers biological specificity. The secretion of HCG by extragonadal tumours is well recognized ${ }^{2}$ particularly by carcinomas of the breast, colon, lung, ovary and stomach. $\alpha \mathrm{HCG}$ and $\beta \mathrm{HCG}$ secretion has been described in up to $70 \%$ of malignant pancreatic endocrine tumours, ${ }^{3}$ and they are considered to be quantitative markers for malignancy. ${ }^{4}$ Rosen et al. ${ }^{5}$ found elevated HCG levels in one third of patients with pancreatic exocrine adenocarcinoma and reported the highest levels in patients with gastric carcinoma. Franchimont et al. ${ }^{6}$ reported elevated serum HCG levels in seminomas (5-22\%) and teratomas $(55-89 \%)$, but fewer than $15 \%$ of breast, gastrointestinal or lung cancers, although a 
proportion (approximately $40 \%$ ) of colorectal carcinomas secrete the hormone. ${ }^{7}$ Metastases from mucinous exocrine pancreatic adenocarcinoma have also been reported to simulate primary tumours of the ovary causing diagnostic difficulties. ${ }^{8}$

Testicular tumours in patients older than 50 years are usually due to metastases. Elevated HCG

\section{References}

1. Haupt, H.M., Mann, R.B., Trump, D.L. \& Abeloff, M.D. Metastic carcinoma involving the testis. Cancer 1984, 54: 709-714.

2. Heyderman, E., Chapman, D.V., Richardson, T.C., Calvert, I. \& Rosen, S.W. Human chorionic gonadatrophin and human placental lactogen in extragonadal tumors. Cancer 1985, 56: 2674-2682.

3. Oberg, K. \& Wide, L. HCG and HCG subunits as tumor markers in patients with endocrine pancreatic tumors and carcinoids. Acta Endocrinol 1981, 98: 256-260.

4. Heitz, P.U., Kasper, M., Kloppel, G., Polak, J.M. \& Vaitukaitis, J.L. Glycoprotein-hormone alpha-chain production by pancreatic endocrine tumours: a specific marker for malignancy. Cancer 1983, 51: 277-282.

5. Rosen, S.W., Weintraub, B.D., Vaitukaitis, J.L., Sussman, H.M., Hershman, J.M. \& Muggia, F.M. Placental proteins and their subunits as tumour markers. Ann Intern Med 1975, 82: 71-83. levels do not necessarily indicate the presence of trophoblastic elements within the tumour. If the metastasis proves to be an adenocarcinoma, raised HCG levels may indicate possible sites for the primary and serve as a marker for disease activity in those patients with malignant pancreatic tumours.

6. Franchimont, P., Collette, J., Hustin, J., Zangerle, P.F., Reuter, A. \& Vrindts, Y. Are chorionic gonadatrophin (HCG) and its alpha and beta subunits useful markers in nontrophoblastic tumours? Ann Endocrinol (Paris) 1984, 45: 235-241.

7. Buckley, C.H. \& Fox, H. An immunohistochemical study of the significance of HCG secretion by large bowel adenocarcinomata. J Clin Pathol 1979, 32: 368-372.

8. Young, R.H. \& Hare, W.R. Metastases from carcinoma of the pancreas simulating primary mucinous tumours of the ovary. Am J Surg Pathol 1989, 13: 748-759. 